\title{
Interacción y comunicación en entornos educativos: Reflexiones teóricas, conceptuales y metodológicas
}

\author{
Marta Rizo García ${ }^{1}$ \\ Universidad Autónoma de la Ciudad de México \\ mrizog@yahoo.com
}

\begin{abstract}
Resumen: El binomio comunicación-educación constituye un ámbito de reflexión que atañe a varios profesionales y científicos sociales, especialmente a los comunicólogos y comunicadores, por un lado, y a los pedagogos, maestros y docentes, por el otro. La mayoría de producción científica en torno a este tema procede del ámbito de la investigación pedagógica y educativa; sin embargo, son cada vez más las aportaciones que se realizan desde las ciencias de la comunicación. Este artículo tiene como propósito reflexionar brevemente en torno a las bases teóricas, conceptuales $y$, en menor medida, metodológicas, que pueden contribuir al desarrollo de conocimiento en torno al campo de la comunicación educativa, especialmente a partir del abordaje de los procesos de interacción que tienen lugar en entornos educativos.
\end{abstract}

Palabras clave: Comunicación, Educación, Interacción, Comunicación Educativa.

\footnotetext{
1 Doctora en Comunicación por la Universidad Autónoma de Barcelona. Profesorainvestigadora de la Academia de Comunicación y Cultura de la Universidad Autónoma de la Ciudad de México y Coordinadora del Plantel Centro Histórico de la misma institución. Investigadora Nivel I, del Sistema Nacional de Investigadores del Consejo Nacional para la Ciencia y la Tecnología (CONACYT, México). Miembro del Grupo hacia una Comunicología Posible (GUCOM), del Grupo de Acción en Cultura de Investigación (GACI) y de la Asociación Mexicana de Investigadores de la Comunicación (AMIC). Co-autora de Cien libros hacia una comunicología posible. Ensayos, reseñas y sistemas de información (UACM, 2005) y autora de La comunicación interpersonal. Introducción a sus aspectos teóricos y metodológicos (UACM, 2005).
} 
Abstract: The binomial communication-education constitutes a scope of reflection that concerns to several professionals and social scientists, especially to the communication researchers and signalers, on the one hand, and to the pedagogues and educational teachers, by the other. Most of scientific production about this comes from the scope of the pedagogical and educative investigation; nevertheless, they are more the contributions that are made from communication sciences. This article has as intention to reflect briefly around the theoretical and conceptual bases, to a lesser extent, methodological, that can contribute to the development of knowledge around the field of the educative communication, especially from the boarding of the interaction processes that take place in educative surroundings.

Key words: Communication, Education, Interaction, Educative Communication.

Resumo: A comunicação-instrução binomial constitui um espaço da reflexão que concerne a diversos profissionais e cientistas sociais, especialmente aos comunicólogos e aos comunicadores, na uma mão, e aos pedagogos, professores educacionais e, pelo outro. A maioria da produção científica sobre esta vem do espaço da investigação pedagógica e educativa; não obstante, são mais e mais as contribuições que são feitas das ciências da comunicação. Este artigo tem como a intenção refletir momentaneamente em torno das bases teóricas e conceituais e, a pouca extensão, metodológicas, que podem contribuir ao desenvolvimento do conhecimento em torno do campo da comunicação educativa, especialmente da abordagem dos processos da interação que ocorrem em arredores educativos.

Palavras-chave: Comunicação, educação, interação, comunicação educativa. 


\section{Aproximación al concepto de comunicación}

En sus acepciones más antiguas, el término comunicación se refería a la comunión, la unión, la puesta en relación y el vínculo. Esta definición se aleja de la visión dominante que asocia la comunicación casi exclusivamente con la transmisión de información a través de los medios masivos. Si las primeras definiciones de comunicación apuntaban a esa dimensión más interpersonal, en la actualidad parece que estas aproximaciones quedaron atrás y son poco tomadas en cuenta en el campo académico.

La comunicación puede concebirse como la interacción mediante la que gran parte de los seres vivos acoplan o adaptan sus conductas al entorno. También se ha entendido a la comunicación como el propio sistema de transmisión de mensajes o informaciones, entre personas físicas o sociales, o de una de éstas a una población, a través de medios personalizados o de masas, mediante un código de signos fijado de forma arbitraria. Y más aún, el concepto de comunicación también comprende al sector económico que aglutina las industrias de la información, de la publicidad, y de servicios de comunicación no publicitaria para empresas e instituciones. Estas tres acepciones ponen en evidencia que nos encontramos ante un término polisémico. Dentro de este abanico de posibilidades, abogamos por una definición que entiende la comunicación como proceso básico para la construcción de la vida en sociedad, como mecanismo activador del diálogo y la convivencia entre sujetos sociales.

La sociedad y la cultura deben su existencia a la comunicación. Es en la interacción comunicativa entre las personas donde, preferentemente, se manifiesta la cultura como principio organizador de la experiencia humana. La comprensión de la comunicación como telón de fondo de toda actividad humana se fundamenta en una perspectiva de corte sistémico, que implica considerar a la comunicación como un conjunto de elementos en interacción donde toda modificación de uno de ellos altera o afecta las relaciones entre otros elementos.

\subsection{Interacción y comunicación}

Los seres humanos establecen relaciones con los demás por medio de interacciones, entendidas como procesos sociales. Al margen de quién o qué inicie el 
proceso de interacción, el resultado es siempre la modificación de los estados de los participantes quienes por el recurso a la comunicación se afectan mutuamente. El enfoque sistémico nos ofrece un punto de partida interesante para abordar el significado del concepto de interacción, y su vinculación con los procesos de comunicación interpersonal. Desde la Escuela de Palo Alto se intentó dar cuenta de las situaciones globales de interacción de las que participa el ser humano. La comunicación fue estudiada como un proceso permanente, no como una situación estática susceptible de ser capturada de forma fija. Esta escuela rompió con la visión unidireccional de la comunicación y abrió las posibilidades para comprender el fenómeno desde una óptica circular. En palabras de Yves Winkin (1994), se pasó del modelo telegráfico al "modelo orquestal de la comunicación": todo cuanto acontece en una situación de interacción adquiere valor comunicativo.

La interacción, además de estar estrechamente relacionada con la comunicación en entornos educativos, se vincula también a los procesos de socialización en general, así como a la manera como los actores de la comunicación construyen su diálogo y se autoerigen en miembros de una comunidad. La socialización supone la capacidad de relacionarse con los demás, de incorporar las reglas del entorno, negociarlas y ajustarlas a sus necesidades. En términos comunicativos, la socialización supone que el sujeto cuenta con los mecanismos necesarios para enviar y recibir información, para interpretarla y significarla. Todo ello, porque el ser humano no se realiza en solitario sino en medio de otros individuos con quienes interactúa.

\section{Comunicación Educativa e interacción}

La Comunicación Educativa parte de la observación de las situaciones comunicativas que se dan en el aula. Si bien gran parte de la producción científica sobre comunicación educativa pone el énfasis en el uso de medios y tecnologías en el aula, estudia su influencia o posibilidades de uso, la reflexión sobre la interacción en entornos educativos presenta un enorme potencial heurístico. Su importancia radica en que nos permite precisar los cambios y las variables más concretas e inmediatas de la experiencia educativa. Este tipo de reflexión complejiza el tratamiento y concepción de la comunicación educativa, al no reducirla a los medios. Mario Kaplun (1983) sintetizó tres modelos básicos de comunicación educativa, que si bien hoy 
podemos tener una perspectiva distinta para analizarlos han sido instrumentos útiles para caracterizar la relación profesor-alumno.

\begin{tabular}{|c|c|c|c|}
\hline $\begin{array}{c}\text { Modelo } \\
\text { Indicador } \\
\end{array}$ & $\begin{array}{l}\text { Énfasis en } \\
\text { contenidos }\end{array}$ & $\begin{array}{l}\text { Énfasis en } \\
\text { resultados }\end{array}$ & $\begin{array}{c}\text { Énfasis en } \\
\text { procesos }\end{array}$ \\
\hline Concepción & Bancario & Manipuladora & Libertadora \\
\hline Pedagogía & Exógena & Exógena & Endógena \\
\hline $\begin{array}{l}\text { Lugar del } \\
\text { educando }\end{array}$ & Objeto & Objeto & Sujeto \\
\hline Eje & Profesor-texto & Programador & Sujeto-Grupo \\
\hline Relación & $\begin{array}{l}\text { Autoritaria- } \\
\text { paternalista }\end{array}$ & $\begin{array}{l}\text { Autoritaria- } \\
\text { paternalista }\end{array}$ & Autogestionaria \\
\hline $\begin{array}{c}\text { Objetivo } \\
\text { evaluado }\end{array}$ & $\begin{array}{c}\text { Enseñar / } \\
\text { aprender (repetir) }\end{array}$ & Entrenar / hacer & $\begin{array}{c}\text { Pensar / } \\
\text { Transformar }\end{array}$ \\
\hline $\begin{array}{l}\text { Función } \\
\text { educativa }\end{array}$ & $\begin{array}{l}\text { Transmisión de } \\
\text { conocimientos }\end{array}$ & $\begin{array}{l}\text { Tecnicas- conductas } \\
\text { Ingeniería del } \\
\text { comportamiento }\end{array}$ & Reflexión-Acción \\
\hline Motivación & $\begin{array}{c}\text { Individual } \\
\text { (premios / castigos) }\end{array}$ & $\begin{array}{c}\text { Individual: } \\
\text { estímulo-respuesta }\end{array}$ & $\begin{array}{l}\text { Comunicación } \\
\text { dialógica }\end{array}$ \\
\hline $\begin{array}{c}\text { Función del } \\
\text { docente }\end{array}$ & Enseñante & Instructor & $\begin{array}{c}\text { Facilitador } \\
\text { Animador }\end{array}$ \\
\hline $\begin{array}{c}\text { Grado de } \\
\text { participación }\end{array}$ & Mínima & Seudo participación & Máxima \\
\hline $\begin{array}{l}\text { Formación de } \\
\text { la criticidad }\end{array}$ & $\begin{array}{c}\text { Bloqueada (o } \\
\text { indiferencia por } \\
\text { parte del maestro) }\end{array}$ & $\begin{array}{c}\text { Evitada (o } \\
\text { indiferencia por } \\
\text { parte del instructor) }\end{array}$ & $\begin{array}{l}\text { Altamente } \\
\text { estimulada }\end{array}$ \\
\hline Creatividad & Bloqueada & Bloqueada & $\begin{array}{l}\text { Altamente } \\
\text { estimulada }\end{array}$ \\
\hline $\begin{array}{l}\text { Manejo del } \\
\text { conflicto }\end{array}$ & Reprimido & Eludido & Asumido \\
\hline $\begin{array}{l}\text { Recurso de } \\
\text { apoyo }\end{array}$ & $\begin{array}{c}\text { Refuerzo } \\
\text { transmisión }\end{array}$ & $\begin{array}{c}\text { Tecnología } \\
\text { Educativa }\end{array}$ & Generadores \\
\hline Valor & Obediencia & Lucro Utilitarismo & $\begin{array}{l}\text { Solidaridad } \\
\text { cooperación }\end{array}$ \\
\hline
\end{tabular}

Fuente: Kaplun (1983: 58).

La realidad social que se vive en la escuela, las interacciones entre los actores del proceso educativo, las cosmovisiones de tales actores, los conflictos, las influencias de la clase social, el lugar en que se vive, las expectativas de vida, la subcultura a la que se pertenece, los conflictos que se desarrollan, las normas que se practican, las creencias, los hábitos, las valoraciones de la cultura ideal y material, las pautas de crianza, las pautas de socialización, en fin, todos los rasgos culturales que 
se dan en la práctica pedagógica constituyen una 'materia prima' que debe ser extraída a fin de comprenderla y transformarla en acción deliberada de los diversos actores implicados en el proceso (Parra, 1998).

\subsection{El Proceso de Enseñanza-Aprendizaje como sistema de interacciones}

Entendemos el proceso enseñanza-aprendizaje como algo complejo que requiere de un proceso de cooperación, producto de la interacción entre los dos sujetos básicos implicados en él: el profesor, instructor, coordinador o facilitador, por un lado, y estudiante, educando, participante, por el otro. La enseñanza tiene como propósito fundamental la transmisión de información mediante la comunicación, sea ésta directa o indirecta o ayudada por soportes o medios auxiliares. Esta acción busca dejar una huella en el sujeto que aprende e influirlo de alguna manera, ya sea en forma de conocimientos o en forma de habilidades y actitudes. El proceso de enseñanza provoca cambios en los individuos, mismos que se dan de forma progresiva y circular; se trata de un proceso progresivo, dinámico, no lineal y con voluntad de intervención y transformación. La enseñanza es un proceso dialéctico, regido por algunas condiciones fundamentales que la hacen posible; se trata de un proceso estrechamente vinculado con la actividad del ser humano, un proceso que condiciona sus posibilidades y aptitudes para conocer, comprender y transformar la realidad de su entorno. La enseñanza se fundamenta en la consecución de ciertos objetivos, entre los que destaca la apropiación de contenidos por parte de los sujetos que aprenden.

Además de ser un proceso, el aprendizaje es también un producto. Aprender es concretar un proceso activo de construcción, es el fruto de una interacción social. Siguiendo a Ileana Alfonso (2003), "un sujeto aprende de otros y con los otros; en esa interacción desarrolla su inteligencia práctica y reflexiva, construye e interioriza nuevos conocimientos o representaciones mentales a lo largo de toda su vida. De esta forma, los primeros favorecen la adquisición de otros y así sucesivamente. De aquí, que el aprendizaje pueda considerarse como un producto y un resultado de la educación y no un simple prerrequisito para que ella pueda generar aprendizajes: la educación devendrá, entonces, en el hilo conductor, el comando del desarrollo”. 
Como resultado de la interacción, el proceso de enseñanza-aprendizaje puede ser considerado como un proceso de comunicación, porque se desarrolla en el marco de relaciones de interacción. Las transferencias de habilidades, conocimientos y actitudes tienen lugar mediante el diálogo o interacción entre facilitadores y estudiantes, y el conocimiento de los estudiantes es juzgado por los docentes a través de juicios que se desarrollan también en un proceso comunicacional.

En términos comunicativos, en el proceso de enseñanza-aprendizaje los facilitadores y los estudiantes son a la vez emisores y receptores de información; producen e interpretan sistemas de mensajes que incluyen palabras, ademanes, gestos, etc. Esta afirmación rompe con la tradicional forma de concebir al estudiante como agente pasivo en el proceso de enseñanza-aprendizaje. En este caso, el estudiante se considera un elemento activo del proceso.

\subsection{Interacción y comunicación en el Aula}

La institución educativa puede abordarse como un sistema abierto, ya que proporciona información hacia el exterior y, a su vez, está marcada por la autoorganización que le permite funcionar hacia el interior, nutriéndose de las interacciones de las que participan los distintos componentes que lo constituyen.

La interacción en el aula se ha abordado desde distintos enfoques y se ha centrado en unidades de observación muy variadas, tales como acercamientos cuantitativos, la relación pedagógica, las relaciones de género, las diferencias en el proceso de enseñanza-aprendizaje por áreas del saber, los factores que propician el éxito o el fracaso escolar y, más en el terreno de lo que nos ocupa, la comunicación, tanto mediada como interpersonal. Este tipo de interacción comprende al conjunto de procesos de relación e intercambio de información que se dan entre los sujetos participantes del proceso de enseñanza-aprendizaje. El intercambio de información no se limita al contenido de los cursos, sino que es más abarcador y toma en cuenta, también, informaciones personales, relaciones de poder, compañerismo, etc. Siguiendo a Fragoso (1999), la comunicación “es más que el maestro habla, el alumno oye. Es más que el simple intercambio de palabras entre personas. Es lo anterior y la manera de expresar, la forma de dirigir el mensaje, el cual tiene dos significados: el 
directo dado por las palabras, y el metacomunicativo, dado por la relación simbólica que se establece entre maestro y alumno".

Desde un enfoque interaccionista, el abordaje de los procesos de comunicación en el aula debe tomar en cuenta el contexto, es decir, las especificidades de la situación en la que se enmarcan dichos procesos. Las interacciones comunicativas en el aula dependen, según Fragoso (1999), “de las reglas que el grupo tiene, de la personalidad del maestro, de su estado de ánimo en ese momento, de cada integrante del grupo, de los líderes del grupo, su personalidad y estado de ánimo, del clima situacional que se dé en cada momento”.

El aula es un espacio social de participación e interacción en el vasto universo escolar, es el lugar privilegiado, por tanto, para la investigación de la interacción en el ámbito educativo. El aula es un espacio de relaciones intrapersonales, interpersonales y grupales, "donde entran en juego los diversos marcos de referencia de las personas que propician muchas veces progreso y otras conflictos" (Fragoso, 1999). Ello comporta que consideremos al aula como un espacio de relaciones, sean éstas de diálogo o de conflicto. El aula es un "un microcosmos compuesto de interacciones conductuales directas, ocurridas entre el profesor y sus estudiantes y entre los estudiantes entre sí. Este microcosmos se amplía en la escuela donde toma la forma de un pequeño sistema social" (Briones, 1990: 50-51). Así pues, si la escuela es un sistema, el aula es uno de sus subsistemas principales, en donde tienen lugar los procesos de enseñanza-aprendizaje, fundamentados en relaciones de interacción entre los sujetos implicados. En el mismo tenor se sitúan las reflexiones de Páez Montalbán (1991: 324), quien define el salón de clases como un "espacio privilegiado y específico para reflexionar sobre la interacción de los actores y convertirse en un ámbito para la observación de los sujetos y de sus complejas relaciones; esto deja de lado discursos y teorías educativas que desconocen lo intersubjetivo son pretexto de la búsqueda de objetividad".

\section{Enfoques teórico-metodológicos para investigar la interacción en entornos educativos}

Durante los últimos años, las reflexiones teóricas en torno a la Comunicación en el Aula han llevado a los educadores y a los comunicadores a 
generar diferentes líneas de investigación acerca de la interacción que existe entre la educación y los factores históricos, culturales, sociales, comunicativos y cognitivos en los que se enmarca el proceso de enseñanza-aprendizaje.

Entre los enfoques que pueden aportar tanto conceptos como estrategias metodológicas para la investigación de la comunicación e interacción en entornos educativos señalamos los siguientes:

- El Interaccionismo Simbólico y su consideración de que por medio de la interacción permanente los sujetos construyen el sentido de las situaciones sociales de la vida cotidiana;

- La Sociolingüística y sus aportes metodológicos para el levantamiento de datos en torno a la comunicación interpersonal por medio de la observación participante, por un lado, y por sus aportes para el análisis del lenguaje utilizado en las situaciones de interacción, por el otro;

- El Constructivismo y sus aportes para la comprensión del procesamiento de información por parte de los actores implicados en la situación de interacción, por un lado, y su abordaje del proceso de enseñanza-aprendizaje como un proceso circular, no lineal, por el otro;

- La Cibernética de Segundo Orden y su propuesta de vigilancia epistemológica que implica el pasar de preguntarnos qué conocemos a preguntarnos cómo conocemos.

Las aproximaciones interpretativas contribuyen significativamente a la investigación en el aula, y esto se da por varias razones, entre las cuales destacan la consideración de las aulas como espacio social y cultural organizado intencionalmente para propiciar el proceso de enseñanza-aprendizaje; su interés en la búsqueda de lo menos obvio, de las relaciones invisibles en las interacciones entre profesores y estudiantes; y, por último, el énfasis dado a la interpretación, significación y construcción de saberes desde el punto de vista de los participantes, propiciando así un enfoque emic -desde dentro- de los objetos/sujetos de estudio. Aplicada en el aula, la investigación de corte interpretativo retoma elementos de la 
ecología social, del contexto o situación objeto del estudio. El investigador procura comprender los modos en los que los facilitadores y los estudiantes, en sus acciones e interacciones conjuntas en el entorno educativo, se modifican unos a otros, en un ambiente de retroalimentación constante.

\subsection{Estudios sobre Comunicación No Verbal}

La importancia del lenguaje no verbal en los procesos de comunicación se pone de manifiesto en la proliferación de investigaciones que lo abordan específicamente en el contexto educativo. El marco teórico para el abordaje de la comunicación no verbal viene dado, fundamentalmente, por los conceptos de kinésica, proxémica y paralenguaje. Edward Hall (1959) propuso algunos elementos básicos para abordar la investigación de la comunicación no verbal en el contexto educativo.

- El investigador debe ser capaz de identificar la identidad individual de los participantes en el proceso de enseñanza-aprendizaje. En este punto, se destacan cuestiones como edad, género, personalidad, empelo, nivel socio-económico, clase, grupo, actitudes sociales.

- Debe exteriorizar la propia capacidad de relación con los otros, es decir, no sólo hay que ver si las relaciones entre maestro y estudiantes es positiva o negativa; sino ver además cómo se perciben y aceptan o enfrentan las diferencias en torno a la autoridad, el poder y la posición social de los protagonistas del acto comunicativo.

- Otro elemento importante viene dado por la percepción de los actos no verbales y los significados que le atribuyen los estudiantes y el maestro, cuando comunican sentimientos y emociones personales. A nivel no verbal, por ejemplo, el rostro puede demostrar miedo, sorpresa, cólera, alegría, etc.

- El investigador debe registrar los movimientos corporales que son señal de determinados sentimientos como la ansiedad. Y asimismo, detectar la capacidad de influencia hacia otros y para uno mismo que logran los participantes en el proceso de enseñanza-aprendizaje. Así, 
se tienen presentes cuestiones como la autoridad, la empatía, la aprobación, la persuasión, etc.

- Por último, el investigador debe registrar el grado de consistencia entre la comunicación verbal y la no verbal, así como identificar el papel del facilitador como moderador del proceso comunicativo.

\subsection{La investigación del discurso en el aula}

Existe consenso sobre la importancia que tiene el habla en el salón de clases, pero no sobre cuál es el mejor modo de estudiar y comprender este lenguaje; las investigaciones concretas sobre el discurso (sobre todo oral) en el aula han sido abordadas desde disciplinas muy diversas, tales como la lingüística, la sociolingüística, las ciencias cognitivas, los estudios socioculturales y socio-históricos, el análisis del discurso, las perspectivas etnográficas o etnometodológicas.

Los estudios del habla en el aula son importantes porque la mayor parte de la enseñanza de los maestros, así como gran parte de las formas en que los estudiantes manifiestan lo que saben, se realiza en el salón de clases por medio del lenguaje, tanto oral como escrito. Es en este sentido que existe una estrecha vinculación entre la investigación sobre los discursos en el aula y los estudios que ponen el énfasis en las interacciones en este mismo lugar: unos no pueden entenderse sin los otros, y a la inversa.

Atendiendo a Candela (2001), los estudios del discurso en el aula desarrollados entre los sesenta y los noventa llegaron a algunos acuerdos metodológicos importantes, que han tenido repercusión en la gran mayoría de investigaciones que se han llevado a cabo en los últimos años. El análisis del habla espontánea; el contexto del habla y su organización social; la relación intrínseca entre el discurso y la acción de los sujetos; la consideración de otros modos simbólicos de representación del mundo, tales como las imágenes, los objetos, los gestos, la percepción del tiempo, entre otros; la importancia otorgada a las reglas de construcción, así como a la normatividad interaccional, que rige toda interacción discursiva; las estructuras de participación que organizan las actividades interactivas; las claves de contextualización que sirven para interpretar los enunciados y las actividades observadas en los entornos estudiados; la definición de la competencia 
comunicativa como la capacidad de leer e interpretar las claves de contextualización; la importancia otorgada a la indexicalidad, que se refiere a que el significado de los enunciados depende del contexto de la interacción discursiva; $\mathrm{y}$, por último, la combinación de estructura y contenido como focos del análisis, son algunas de las repercusiones metodológicas del enfoque de los estudios del discurso en el aula.

\subsection{La etnografía educativa}

La etnografía es un método de investigación mediante el cual se aprende el modo de vida de una unidad social concreta. A través de ella se persigue la descripción o reconstrucción analítica de carácter interpretativo de la cultura, las formas de vida y la estructura social del grupo investigado. Según Atkinson y Hammersley (1994) la etnografía otorga un fuerte énfasis a la exploración de la naturaleza del fenómeno social concreto. La etnografía en el entorno educativo debe partir de la siguientes preguntas: ¿Qué sucede en el entorno educativo?, ¿Qué hacen los sujetos estudiados en ese entorno?, ¿Qué significa para la gente involucrada aquello que sucede en el entorno?, ¿En qué forma la organización de patrones de relación es similar o diferente a la que se da en otros lugares?

Los estudios etnográficos se centran en investigar un pequeño número de casos, quizás uno solo, pero en profundidad. De sus requisitos más importantes destacan la realización de observación directa, la mayoría de veces participante, la permanencia temporal extensa en el escenario objeto de estudio, el gran volumen de datos registrados y el carácter evolutivo, procesual y dinámico del estudio etnográfico.

\subsection{Etnografía de la comunicación}

La etnografía de la comunicación busca entender cómo el conocimiento social, psicológico, cultural y lingüístico determina los usos del lenguaje. En los años sesenta, Hymes (1972) desarrolla su propuesta de etnografía de la comunicación. Una década más tarde se da el auge de la etnometodología, perspectiva desde la que se inauguran importantes estudios sobre el discurso comprendido como acción social. En los ochenta y noventa se concibe al lenguaje como uno de los modos que, en 
interacción con otras formas, permiten conocer la representación de conocimientos y estados mentales y la comunicación en contextos educativos.

En términos generales, la etnografía de la comunicación describe los patrones de interacción de los miembros de un grupo cultural determinado. Uno de sus supuestos de partida básicos es que tanto la comunicación verbal como la no verbal son formas culturalmente condicionadas. En cuanto a las metodologías, la etnografía de la comunicación ha utilizado fundamentalmente la observación participante, casi siempre acompañada por grabaciones de video y audio de las interacciones verbales y no verbales, que son "capturadas" en su entorno natural.

Hymes presenta un modelo para el análisis de interacción, donde entran en juego varios elementos: la situación se entiende en el sentido de localización espacial y temporal en que se produce el encuentro comunicativo; los participantes son quienes intervienen, con sus características socio-demográficas, su repertorio verbal y su bagaje cultural particulares; las finalidades se refieren tanto a los objetivos como a las metas de la interacción como en los productos que se obtienen y puede coincidir o no con las finalidades; las secuencias de actos se refieren a cómo se estructura y organiza lo que se va diciendo en la interacción, es la manera como los temas se presentan, se desarrollan o cambian; la clave es el grado de formalidad / informalidad de la interacción; los instrumentos incluyen el canal o medio a través del cual circula el mensaje; las normas en la interlocución (de forma tanto explícita como implícita) regulan la toma de la palabra; y por último, el género atiende al tipo de hecho comunicativo (sesión de tutoría, sesión de estudio, conferencia, clase de un profesor invitado, junta de trabajo, etc.) que puede darse en un entorno educativo.

\subsection{Estudios etnometodológicos}

La etnometodología se centra en el análisis de las actividades prácticas cotidianas, dotándolas del mismo interés que en gran parte de la sociología estándar se ha dado a eventos aparentemente más importantes. Su creador es H. Garfinkel (1967), quien a partir de los años setenta incorporó la importancia del análisis conversacional centrado en la organización del diálogo en la actividad cotidiana y en cómo se presenta la ordenación y coherencia en los intercambios conversacionales. 
El foco de interés son las personas en su interacción cotidiana y las actividades que desarrollan en sus contextos inmediatos; por eso se sitúa en el ámbito de la microsociología o sociología de la situación. Los estudios etnometodológicos dirigen su atención hacia las actividades diarias en las que nos vemos envueltas las personas; estas actividades son concebidas y analizadas como métodos a los que las personas recurrimos para hacer que estas actividades sean explicables.

En el terreno concreto de las investigaciones en entornos educativos, la Etnometodología puede contribuir a investigar asuntos como la organización del diálogo entre profesor y estudiantes, y entre estudiantes y estudiantes; la importancia que otorgan los estudiantes a las actividades propuestas por el profesor; y el significado global que los estudiantes le dan al proceso de aprendizaje en el que están inmersos, entre otros aspectos.

\subsection{La Investigación-Acción-Participativa}

En la formación y desarrollo del profesor se aplica la metodología de investigación-acción (IAP) participativa, en la que el campo de investigación es demarcado por los profesores integrantes y de esta manera actúan crítica, participativa y cooperativamente en él. La IAP implica "comprensión, control crítico y sucesivas reconstrucciones, base de actuaciones posteriores" (Medina et. al., 1993: $60)$.

En ella se identifican algunos elementos que la convierten en una forma de construcción de conocimientos muy particular. La IAP tiene un propósito eminentemente transformador, de intervención en la realidad social. En la aplicación de esta técnica, el investigador determina el clima de participación de los miembros del grupo, de modo que todos toman decisiones, seleccionan las áreas de estudio y las propuestas de análisis. Esta afirmación, en términos de la investigación educativa, adquiere gran importancia, dado que la mayoría de estudios enfocados a las interacciones en entornos educativos no se desarrollan con el fin único de construir conocimientos, sino que se proponen, como finalidad fundamental, intervenir y modificar la realidad estudiada. 


\section{Cierre sintético}

En este texto se han puesto de manifiesto las posibilidades que presenta el campo de investigación de la Comunicación Educativa. Sobre todo hemos querido dejar a un segundo plano aquellas aportaciones que se centran casi exclusivamente en los medios de difusión masiva, o las nuevas tecnologías, y hemos dado mayor importancia a la corriente de estudios de la interacción en el aula. Como se ha podido ver, este campo de interés abarca numerosos conceptos y requiere de una mirada multidimensional acorde con su objeto de estudio. Asimismo, las estrategias metodológicas posibles para los análisis de la interacción y comunicación al interior del aula son numerosas y requieren de la triangulación metodológica en aras de construir conocimientos más abarcadores y completos.

\section{Referencias bibliográficas}

Alfonso Sánchez, Ileana (2003) "Elementos conceptuales básicos del proceso de enseñanza-aprendizaje", en Acimed. Revista cubana de los profesionales de la información y la comunicación en salud, Núm. 6, Vol. 11, noviembrediciembre 2003. Artículo en línea, disponible en http://bvs.sld.cu/revistas/aci/vol11 6 03/aci17603.htm (Fecha de consulta: octubre 2006).

Atkinson, P. y Hammersley, R. (1994) "Ethnography and Participant Observation", en Denzin, N. K. y Lincoln, Y. S. (eds.) (1994) Handbook of Qualitative Research, Londres: Sage Publications.

Briones, Guillermo (1990) La investigación en el aula y en la escuela, Bogotá: Andrés Bello.

Candela, Antonia (2001) "Corrientes teóricas sobre discurso en el aula”, en Revista mexicana de investigación educativa, Vol. 6, Núm. 12, mayo-agosto 2001. Artículo en línea, disponible en http://www.comie.org.mx/revista/Pdfs/Carpeta12/12invest1.pdf (Fecha de consulta: octubre 2006).

Fragoso Franco, David (1999) “La comunicación en el salón de clases”, en Razón y palabra, Núm. 13, Año 4, enero-marzo 1999. Artículo en línea, disponible en 
http://www.razonypalabra.org.mx/anteriores/n13/comsal13.html (Fecha de consulta: octubre 2006).

Garfinkel, Harold (1967) Studies in Ethnomethodology, New York: Prentice-Hall.

Hall, Edward T. (1959) The Silent Language, Nueva York: Doubleday.

Hymes, Dell (1972) Functions of Language in Classroom, Nueva York: Teachers Colleges Press.

Kaplun, Mario (1983) El comunicador popular, Buenos Aires: Humanitas.

Medina, Antonio y Domínguez, M. Concepción (1993) Enseñanza y currículum para personas adultas, Madrid: EDIPE.

Páez Montalbán, Rodrigo (1991) "El salón de clase, un mundo de sujetos”, en M. Rueda, G. Delgado y M. A. Campos (1991) El aula universitaria. Aproximaciones metodológicas, México: UNAM.

Parra, Maria Eugenia (1998) "La etnografía de la educación”, en Cinta de Moebio, Núm. 3, Abril 1998. Facultad de Ciencias Sociales, Universidad de Chile. Artículo en línea, disponible en http://www.moebio.uchile.cl/o3/frprino4.htm (Fecha de consulta: octubre 2006).

Winkin, Yves (ed.) (1994) La nueva comunicación, Barcelona: Kairos. 